\title{
Configuración de redes sociales en personas mayores viudas en Chile
}

\author{
Paulina Osorio-Parraguez \\ Doctora en Sociología (Universidad del País Vasco) \\ Profesora en la Universidad de Chile \\ Santiago de Chile, Chile \\ posorio@uchile.cl
}

Andrés G. Seguel

Doctor en Sociología (Universidad del País Vasco)

Profesor en la Universidad de Chile

Santiago de Chile, Chile

angomez@uchile.cl

Pamela Jorquera

Estudiante de doctorado en Antropología Social (Universidad Federal de Rio Grande del Sur) Profesora en la Universidad de Chile

Santiago de Chile, Chile

pjorquera@facso.cl

Resumen El artículo presenta los resultados de una investigación sobre la configuración de redes de relaciones sociales, en personas viudas mayores de 60 años, en la Región Metropolitana de Santiago, Chile. Este trabajo apunta a la conjunción entre estructuración del campo de la vejez y las experiencias vitales de las personas mayores. A través de una investigación acotada a los procesos de envejecimiento en Chile, se observan - a través de una metodología de análisis de redes sociales -, la morfología de las redes y tipos de vínculos de las personas mayores viudas antes y después de enviudar y cómo ello influye en la construcción social de la vejez; en el fondo, cómo se construye y reconstruye la identidad del envejecido al entender la muerte del cónyuge como un hito biográfico-social, siendo las relaciones interpersonales, su construcción, experiencia y significaciones, los ejes para el análisis y comprensión del fenómeno social de la viudez en la vejez.

Palabras clave: vejez, viudez, relaciones interpersonales, transiciones biográficas.

\section{Introducción ${ }^{1}$}

A ctualmente asistimos a un profundo cambio demográfico caracteA rizado por el envejecimiento acelerado de la población mundial, es decir, la disminución de las tasas de natalidad frente a un aumento significativo de las expectativas de vida. Chile, al igual que otros países de América Latina, ha experimentado un envejecimiento sostenido de su población. En el año 2010, el $13 \%$ de la población correspondía a personas de 60 años y más, proyectando para el 2050 un aumento

1. Trabajo realizado en el marco del proyecto Fondecyt n. 1110110 "Procesos estructurales de la viudez en la construcción social del envejecimiento". Los autores agradecen la colaboración para la preparación de este artículo a la magíster Cynthia Meersohn, a la socióloga, Javiera Araya y a la licenciada en Antropología Social, Catalina Careaga. 
del 28,2 \% (INE, 2002). Del total de la población en Chile, el 50,9\% son mujeres, y, en el caso de la población mayor de 60 años, esa cifra aumenta al 56,3\%.

El envejecimiento, como campo de estudio de las ciencias sociales y la gerontología, abarca al menos tres dimensiones que están directamente asociadas a las dinámicas de las poblaciones envejecidas: una dimensión aborda la configuración de sociedades cada vez más envejecidas, es decir, un fenómeno asociado directamente a la demografía, la pirámide poblacional y los efectos en la organización y estructura social; otra, la afectación de las personas envejecidas y su significado psicosocial; y por último, la que se ocupa de los procesos de envejecimiento asociado a la forma en cómo se organiza el envejecimiento, ocupando un lugar preponderante el efecto de la estructura social en este proceso.

Es en esta última dimensión donde los elementos estructurales de la sociedad y las experiencias vitales de las personas mayores se conectan; es por ello que el énfasis esta puesto en la viudez como punto de inflexión cultural del proceso de envejecimiento, y su capacidad como factor condicionante de la estructuración social del envejecimiento será abordada a través de la configuración de redes sociales.

\section{La vejez y la viudez como objetos de investigación social}

En el campo de estudio referido a la vejez, el acercamiento que se ha hecho desde las ciencias sociales ha estado determinado por las múltiples transformaciones que se producen en las personas a lo largo de la vida, tanto físicas, como económicas y sociales. Por lo mismo, no existe un paradigma único para conceptualizar a la vejez y menos aún al envejecimiento. Su carácter multidimensional como fenómeno social ha provocado que su observación determine varias perspectivas de análisis que utilizan, por un lado, a la edad como punto de partida; por otro, al sistema o estructura social; y por otro, al envejecido y su interacción social y la de las instituciones que le circundan.

A partir de los años ochenta comienza un mayor interés de la teoría social frente a los estudios de envejecimiento, principalmente con el desarrollo de la llamada economía política de la vejez y gerontología crítica. Este nuevo enfoque -que se construye sobre la base de diversos discursos- significa un aporte al conocimiento del proceso de envejecimiento y de la vejez en cuanto construcción social y más allá que un fenómeno psicobiológico. Este desafío a la tradicional perspectiva en gerontología abarca desde cuestiones prácticas de políticas y el papel del Estado en la gestión de la vejez hasta cuestiones teóricas del envejecimiento.

Posteriormente, "la perspectiva postmoderna del envejecimiento analiza la vejez como una dimensión importante del cambio social [...]. Surgen así múltiples estilos de vida que ya no se basan en las productividad sino en el consumo [...]; se produce una tendencia creciente a abandonar el modelo de vejez como patología social y surgen aproximaciones teóricas emancipatorias que tienen en cuenta más la posibilidad de dar poder a las personas" (Bazo, 2001, p. 22). En un contexto de conocimiento, estudio y análisis social del envejecimiento y la vejez, surge con fuerza la cuestión de dotar de poder a las personas o empowerment, junto a la preocupación por la transformación de la sociedad y el desarrollo de nuevos rituales y símbolos en torno a los cambios del curso de la vida. Al no ser todos los conceptos asociados a la vejez aplicados de la misma forma en cuanto a la cultura, género y clase social, las perspectivas feministas en envejecimiento y el enfoque cultural de la diversidad en envejecimiento, también amplían los planteamientos de la gerontología crítica (Osorio, 2006).

Dentro de esta última perspectiva, emerge también el enfoque del envejecimiento productivo y el que entiende la edad como una producción social, en el se propone un marco conceptual que la comprende, siguiendo a Bourdieu, como un campo. En este sentido, la propuesta de Gutiérrez y Ríos (2006) enmarca el quehacer científico e investigativo de la gerontología social en el "contexto" de las "edades", al entenderlo como un espacio y una referencia cultural mucho más apropiada para el estudio del envejecimiento y la vejez.

Se observa a la vejez integrando las relaciones que guarda con las demás edades, y es en ese haz de relaciones y significaciones producidas social e históricamente que se delimitan unidades y categorías de estudio, vale decir, se entiende que las edades -y en ellas la vejez- también se producen en torno a una serie de prácticas sociales que buscan categorizarlas y significarlas, construyendo un espacio social determinado en un proceso histórico particular.

Otra perspectiva que ha abordado el proceso de envejecimiento es la sociopoiética, es decir la observación del envejecimiento y la vejez a partir de la distinción entre inclusión y exclusión social, caracterizando los diferentes niveles y sistemas en los que se pueden observar dichos fenómenos, y, lo más relevante para este artículo, entendiendo a las redes sociales como un elemento de inclusión de las personas mayores (Arnold et al., 2009).

En el presente artículo, se observa el fenómeno del envejecimiento y la vejez desde el paradigma del curso de la vida y desde una perspectiva socioantro- 
pológica. Al entenderlos como una experiencia social, emergen una serie de dimensiones que intervienen en su construcción, a saber, la edad; la valencia/ dependencia; la jubilación; y las transformaciones de las redes, roles y relaciones sociales de la personas al envejecer. Estas han destacado en el campo de análisis de los procesos sociales de envejecimiento enfatizando su carácter e interés sociocultural.

En este marco, el factor viudez como factor de estructuración social no ha sido investigado en directa relación con la vejez y el envejecimiento. La viudez, si bien es una experiencia experimentada por las personas en cualquier momento de sus vidas, puede tener una característica específica en el caso del proceso de envejecimiento, esto es, la de desplegar la estructura social, pues ante la falta del cónyuge, se recomponen los vínculos sociales y ocurren una serie de cambios significativos en la experiencia cotidiana y biográfica del sujeto que envejece. Es uno de los elementos que les permiten constituir su identidad de envejecientes. Así, la viudez puede significar un cambio estructural significativo, que activa un rol diferente de articulación consigo mismo y con los otros. Ser viuda o viudo implica más que la sola muerte del cónyuge; es una categoría cultural que se adquiere al cumplir una serie de requisitos, y solo uno de ellos es la muerte del marido o la esposa (Buitelaar, 1995).

\section{Redes sociales, envejecimiento y viudez}

Las redes y roles sociales, considerados como categoría de análisis, hacen referencia a los significados y a la transformación, pérdida y adquisición de relaciones sociales, que el proceso de envejecimiento implica, en este caso como resultado de la viudez ya que muchas de las redes sociales y el contacto social de las personas mayores se encuentran mediados por el estado marital (Scott y Wenger, 1996).

Tanto la teoría del intercambio como la teoría de la afinidad en envejecimiento afirman que las relaciones conyugales de rol en hombres y mujeres deben ser más simétricas. La teoría de la afinidad establece una relación directa entre roles desempeñados y conductas. De tal forma que, a mayor similitud entre los roles desempeñados entre hombres y mujeres, las conductas serán mayormente semejantes. La teoría del intercambio también supone una relación de simetría y reciprocidad, de modo que "las características de cada cónyuge tendrían un efecto similar en la pauta [...] de su compañero o compañera, con independencia del género" (Arber y Ginn, 1996, p. 109). La dimensión de género ha sido destacada por varios autores como necesaria para el estudio de las trayectorias biográficas y generacionales (Del Valle, 2002; Moen y Spencer, 2006; Ingrisch, 1996; Bernard et al., 1996), y para acercarnos a la temática de las relaciones conyugales y familiares durante la vejez (Askham, 1996; Wilson, 1996; Rose y Bruce, 1996). Desde una perspectiva funcionalista se ha considerado norma social que la mujer dependa económicamente de su marido a lo largo de la vida y con mayor razón durante la vejez, y que ella asuma la responsabilidad de los cuidados y afectos en el interior de las relaciones conyugales y familiares. Las personas casadas tienden a mantener relaciones casi exclusivamente con familiares y personas muy cercanas y su nivel de contacto social es escaso, incluso durante el periodo previo a la muerte de uno de los cónyuges y en que se encuentra enfermo/a. Al enviudar, el contacto social se incrementaría casi inmediatamente y produciría una mayor variedad en el tipo de relaciones sociales debido al cambio que significaría la viudez, aumentando las redes sociales de apoyo; sin embargo, al cabo de pocos años, el contacto y las redes sociales de los viudos y viudas se reduce nuevamente a los niveles que tenía antes de pasar por esta transición (Guiax et al., 2007). Los estudios lo corroboran, mostrando que el matrimonio llega a ser la relación central para muchos adultos, pues los provee de redes sociales y actividades de ocio (Patterson y Carpenter, 1994), por ello, la muerte del cónyuge y la consecuente viudez sería una situación que les afecta no sólo emocionalmente, sino que también afecta a todo su sistema de relaciones interpersonales y a la configuración de su red social (Berardo, 1970, p. 13; McCallum, 1986). Las personas viudas experimentarían un ajuste en sus relaciones, cambiando su estatus dentro de la familia y la sociedad (Ferraro, 1984), con la viudez como el umbral hacia una serie de transiciones y cambios en sus vidas y vínculos.

La viudez, para el caso de los hombres, puede representar la pérdida de relaciones íntimas (Wister y Strain, 1986), ya que habría menos viudos que viudas con los que se pueda entablar amistad. Además, las redes sociales fuera del trabajo han estado más orientadas hacia las mujeres que hacia los hombres, por lo que ellas tendrían más posibilidades de encontrar apoyo en amigos y vecinos que los viudos (Swann, 1988). Luego de enviudar y durante un periodo de ajuste, los hombres recurrirían a las amistades para proveer el apoyo emocional que solían recibir de su esposa. A medida que pasa el tiempo estas amistades se fortalecen o debilitan, y aquellas que duran más tiempo pueden ser alteradas por la enfermedad o la muerte. Aún varios años después de la muerte de su esposa, siguen manteniendo la sensación de pérdida, pero con una actitud orientada a acostumbrarse a la soledad. Si bien las amistades reducen la sensación de soledad, el nivel de compromiso al que los viudos lle- 
gan en este tipo de relaciones es limitado. Los que encuentran nuevas parejas prefieren socializar en lugares públicos, manteniendo a sus nuevas compañeras fuera de su espacio privado y centrando sus actividades en cosas como salir a comer, que resulta en oposición a "comer solo" (Riggs, 1997).

La brecha entre hombres y mujeres que vuelven a casarse luego de enviudar no se debe exclusivamente a la actitud frente a este estado, aun cuando exista la creencia social de "las mujeres lloran, los hombres reemplazan", sino que tiene que ver también con una tendencia demográfica a nivel mundial, ya que las mujeres viudas son muchas más que sus pares masculinos. ${ }^{2}$ A pesar de que los viudos tienden a casarse más que las viudas, el matrimonio después de la viudez no es una tendencia contrastada. Aun así, las cifras oficiales tienden a subrepresentar el interés en relaciones sentimentales, ya que hay muchas personas viudas, especialmente mujeres, a las que les gustaría volver a casarse, pero no lo hacen debido a obstáculos demográficos, financieros o normativo-culturales. Las dinámicas maritales suelen poner al hombre como el que está en control de las cosas y las mujeres como las compañeras cuidadoras y empáticas. Una vez que muere el cónyuge, los hombres muestran mayor inclinación que las mujeres a buscar pareja, mientras que ellas se sienten liberadas de ese rol de cuidadoras, aunque a la vez se sienten culpables de ello. Las mujeres mayores tienden a asociar una nueva relación con ataduras, mientras que los hombres, a pesar de estar socializados en la independencia, buscan a una nueva compañera que los cuide (Davidson, 2001).

Con el paso a la viudez se producen cambios en las interacciones dentro de la red social, cambios que pueden tender a su aumento o disminución. En las mujeres las redes sociales son más amplias cuando necesitan ayuda o asistencia, especialmente en aquellos casos donde se ha enviudado recientemente. Al quedar viudas atraerían mayor atención y preocupación de sus redes. En algunas viudas se produce una sensación de liberación al morir el cónyuge, sobre todo en las que cumplieron la función de cuidadora de su marido; al fallecer el marido aumentarían sus actividades y relaciones sociales (McLaughlin et al., 2011).

En la vejez, la viudez - como factor de estructuración social- adquiere un carácter relevante, pues es uno de los elementos que está presente en la experiencia de envejecer, sobre todo en las mujeres. Así, la viudez, como ya se ha mencionado, ha aparecido como una realidad principalmente femenina cuando la cruzamos con la vejez o la edad mayor.

La investigación realizada se fundamentó en el hecho de que al observar la condición de viudez en la vejez, no solo se nos muestra a una persona que ha perdido a su cónyuge, sino también lo que ser hombre o mujer viejo/a significa en un continuo de experiencia vital que también conlleva cambios claves para comprender el proceso de envejecimiento en las personas. Se aborda de esta forma una aproximación a una definición de vejeces distintas en hombres y mujeres mayores viudos/as.

\section{Metodología}

La investigación es del tipo descriptivo y explicativo, y se enmarca dentro de una aproximación mixta, que utiliza técnicas de producción de información cualitativas y cuestionarios de redes para el conocimiento de la configuración de las redes de relaciones sociales e interpersonales en personas mayores antes y después de enviudar. La técnica cualitativa utilizada fue la entrevista en profundidad de carácter biográfico; se realizaron preguntas abiertas que permitieron al propio entrevistado ir estructurando la dinámica y la construcción de su discurso, permitiendo obtener una visión global y compleja de sus vidas, de sus relaciones sociales y acceder de manera más amplia al conocimiento de la forma cómo sus narradores significan sus relaciones, sus vínculos personales y asumen las transiciones y cambios individuales, familiares y sociales que la viudez en la vejez conlleva. Se analizaron relatos de relaciones interpersonales, trayectorias conyugales y de viudez, y en base a ello se construyó un cuestionario para conocer el tamaño y la estructura de la red de ego antes y después de enviudar; este contó con tres ítems: (i) definición del tipo de redes que estudiar: redes egocéntricas o personales; (ii) definición de la tabla de atributos: identificación de actores con contacto regular antes y después de la viudez; (iii) definición de tablas de adyacencia: relaciones existentes entre los actores identificados.

La investigación siguió las orientaciones de un muestreo teórico y de tipo intencional, en la cual se fueron decidiendo los datos que se iban produciendo y en dónde encontrarlos. La muestra teórica se basó en la selección de sujetos desde sus características estructurales y que remiten a su posición en una cierta estructura, que dieron forma a los criterios de selección de la muestra en base a la definición de variables de acuerdo con los objetivos de la investigación. Se optó por una muestra intencionada que permitió conocer, descubrir y describir el fenómeno estudiado en profundidad. También se consideró a la hora de defi- 
nir la muestra el criterio de calidad de la información, por lo tanto, su conveniencia y pertinencia.

La muestra estuvo compuesta por 36 personas mayores que enviudaron después de los 60 años y que llevan por lo menos un año de viudez.

El corpus de información obtenida fue sistematizado con el software para el análisis de redes sociales UCINET 6, para el posterior proceso de interpretación de los datos. Se observó y comparó la composición de la red social en el periodo antes de la viudez con el periodo después de la viudez, buscando constituir a la viudez como un hito relevante en la configuración de las redes sociales en el análisis realizado. Siguiendo ese objetivo se procedió también a una caracterización de los atributos de las redes antes y después del hito de la viudez, para, posteriormente, analizar las propiedades estructurales de las redes. Por medio de estas, es posible observar las características de las redes, ver los niveles de integración que poseen, la presencia o no de subestructuras y el nivel de influencia de algunos de sus miembros.

Para los casos estudiados se observó la pertinencia de analizarlos en términos de densidad, propiedad que mide el nivel de integración de la red en base a la proporción de relaciones existentes sobre el total de relaciones posibles de una red social determinada. Además se revisaron las medidas de centralidad, que permite saber cuáles son los actores más influyentes, prominentes o poderosos de la red, y de intermediación, que permite identificar cuáles son los actores que relacionan a los contactos entre sí y por tanto facilitan el acceso entre ellos (Herrero y Sánchez, 2000).

\section{Resultados y análisis de la información empírica}

\section{Actores y sus características en redes de mujeres antes de enviudar}

Las redes sociales de las mujeres analizadas se caracterizan por estar constituidas mayoritariamente por actores o contactos de tipo familiar (cónyuge, hermanos, hermanas, cuñados, cuñadas, hijos, hijas, nietos, nietas) y por amigos y amigas, siendo el vínculo familia el que tiene mayor presencia en la configuración de las redes. Con respecto al género de los contactos establecidos por las mujeres, en este periodo, estos son principalmente mujeres, existiendo en menor medida la presencia del género masculino. En los casos en que existen componentes masculinos, estos forman parte del grupo familiar (cónyuge, hermanos, hijos, nietos). También se observa, en menor medida, la presencia de algunas vecinas en las redes sociales de los casos de las mujeres estudiadas, algunas personas con las que comparten algún tipo de actividad -cursos, espacio laboral- los que hemos identificado como compañeros y compañeras, y algunos contactos que son más que amigos, pero no son familiares a los que denominan compadres y comadres. ${ }^{3}$

\section{Actores y sus características en redes de mujeres después de enviudar}

Después de enviudar, las redes sociales de las mujeres entrevistadas mantienen algunas características, pero también manifiestan cambios en su morfología. Esas características se asocian al número de contactos, tipos de vínculos, presencia de nuevos contactos y actores que desaparecen de la red. A continuación describiremos la composición de las redes sociales estudiadas identificando los elementos que se mantienen, así como los cambios que se producen con la viudez.

La primera característica que destaca al analizar las redes sociales de las mujeres entrevistadas después de enviudar es la reducción de los contactos producto del fallecimiento de algunos actores, principalmente de los contemporáneos, dada la condición de vejez de los casos estudiados. La segunda es que, pese a la disminución de actores, la mayoría de las mujeres incorporan actores nuevos a la red, los que se integran principalmente en el ámbito de los cuidados y de nuevas actividades. La tercera es que los vínculos que establecen las mujeres corresponden mayoritariamente a familiares, y, finalmente, en relación al género de los integrantes de la red social, en este periodo, es también prioritariamente femenino; en ese sentido, las mujeres reducen los contactos con el género masculino.

En general se observa que la estructura principal de las redes sociales se mantiene ya que todas se concentran en contactos de tipo familiar. Sin embargo, van desapareciendo los familiares contemporáneos, tomando un lugar más protagónico los descendientes (hijos e hijas, yernos y nueras, nietos y nietas). Por ejemplo, en algunos casos, el vínculo familiar nuera llega a adquirir mayor importancia dentro de los vínculos familiares.

La ausencia de algunos actores de la red previudez modifica la morfología de la red posviudez de las entrevistadas. Si bien con la incorporación de nuevos actores, el número de ellos se mantiene, los vínculos entre los contactos se reducen considerablemente, es decir, ahora se tiene menos contactos a quienes poder

3. Independiente de si es padrino o madrina de alguno de los hijos, es costumbre llamar compadre o comadre a aquellas personas con quienes se tiene un vínculo de amistad muy cercano, que llegan a ser consideradas como un miembro de la familia sin serlo. 
recurrir frente a un problema, lo que ubica a algunos integrantes de la red posviudez, generalmente a la hija, en una posición gravitante ya que muchas veces de ellas depende el cuidado de la entrevistada. En otros casos el lugar ocupado por el cónyuge -y por tanto los roles y funciones desempeñadas- es asumido por el hijo, produciéndose un intercambio en términos de roles y funciones en la red social de las mujeres viudas. Por ello, la red mantiene sus ejes principales pero se recompone ante la ausencia de algunos contactos centrales (véase la figura 1).

Destaca también la incorporación de nuevos tipos de vínculos a las redes posviudez, como es el caso de la figura del cuidador, que siempre es una mujer. Estos vínculos generan una subestructura en la red social de las mujeres viudas, las que mantienen vínculos entre sí y comparten la cotidianeidad con la entrevistada; no obstante, esos actores no mantienen vínculos con la estructura principal de la red, lo que las convierte en redes frágiles pese a su importancia en la vida cotidiana de la viuda.

En otros casos se observa que con el paso a la viudez la red social varía en su configuración, producto del fallecimiento de sus actores principales, quedando solo un vestigio de la red previudez. En estos casos se presenta una nueva red formada por contactos nuevos, correspondientes a los vínculos de tipo compañeros y compañeras. En ellas la figura de las compañeras de la red previudez que no tenían vínculos con la estructura principal de la red y que, por lo tanto, asumían un rol periférico, en la red posviudez asumen un rol central relacionando a diversos actores (véase la figura 2).

Finalmente, destacan casos en donde la red previudez se desintegra totalmente por la muerte del cónyuge, quien cumplía la función de conector entre sus diferentes integrantes; con su ausencia no hay quien relacione a los actores, puesto que su rol no es asumido por algún otro contacto. Esta desintegración de la red produce su pérdida de densidad, reduciéndose los contactos de la viuda a vínculos principalmente familiares.

\section{Propiedades estructurales de las redes sociales de mujeres}

En las mujeres, las redes sociales previudez corresponden a redes densas, ya que los actores mantienen algún vínculo con la red, ya sea de forma directa o intermediados por otros; en ese sentido se observa la inexistencia de contactos aislados, sin vínculos con los demás actores que forman la red. Además, en las redes sociales de las viudas se advierte un alto nivel de centralidad y de intermediación, lo que quiere decir que algunos de los contactos presentes en la red son más influyentes o poderosos que el resto ubicado en la periferia. De las redes sociales revisadas, los actores ubicados en esas posiciones corresponden a vínculos de tipo familiar directo (familia nuclear) donde destaca la figura del cónyuge.

En las redes sociales postviudez se observa que la densidad se mantiene en niveles muy parecidos, es

\section{MUJER}

FIGURA № 1: “Configuración de red social en mujer antes y después de enviudar, según centralidad”
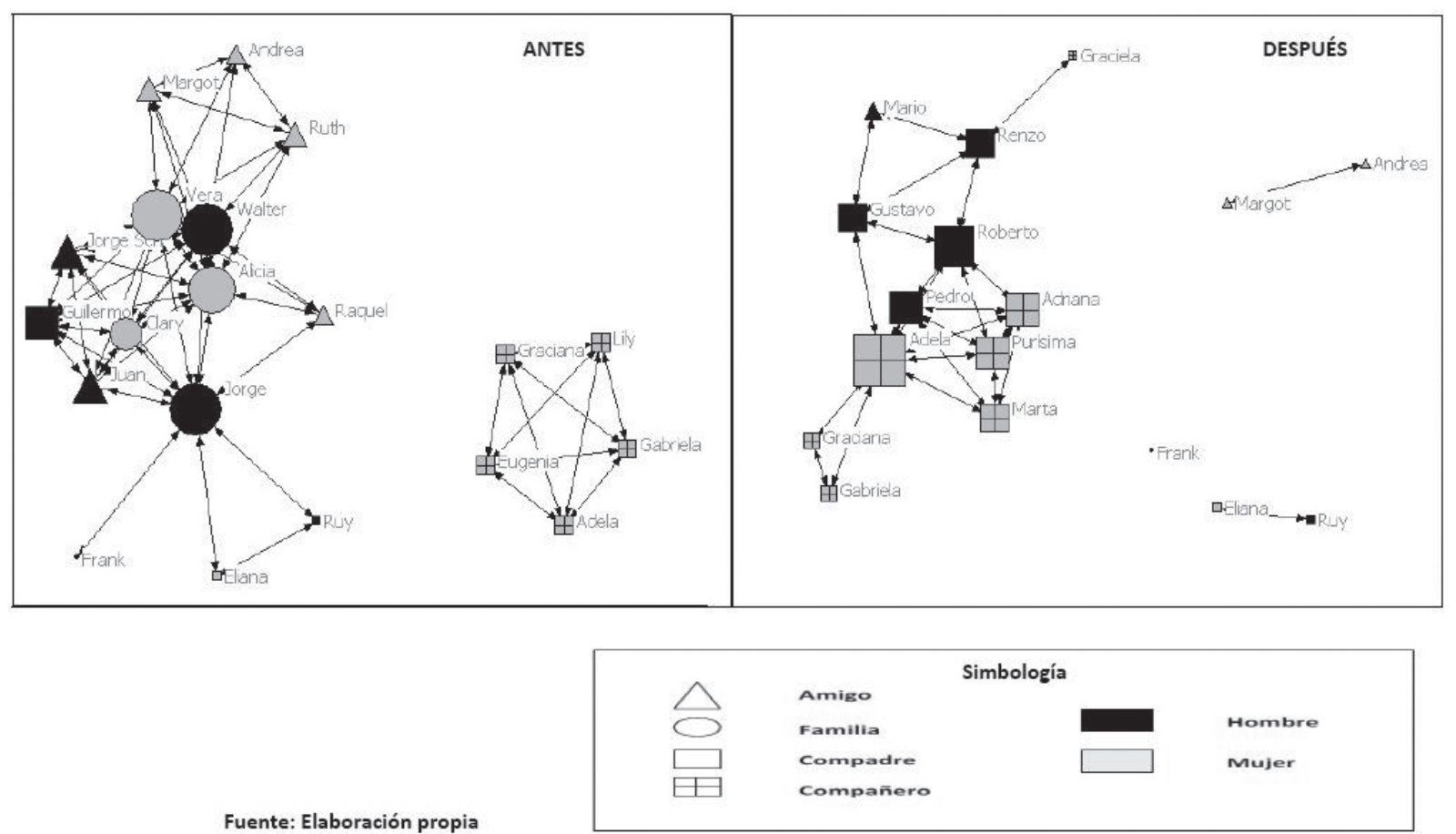
decir, que los actores siguen estando vinculados entre sí (por ejemplo 0,5 antes y 0,4 después de enviudar), pero las medidas de centralidad e intermediación bajan (por ejemplo en centralidad 30,5 \% a 17,1\%; e intermediación $56,4 \%$ a $28,6 \%$ ), lo que implica la desaparición de un actor importante que cumplía esas funciones anteriormente, siendo ocupadas esas posiciones por nuevos contactos. En algunos casos, esas posiciones son ocupadas por un solo actor, principalmente los hijos, lo que muestra una gran responsabilidad sobre ese actor en la mantención de la red social de la viuda. En los casos donde la red previudez se modifica totalmente o se desintegra, las mujeres viudas son capaces de construir nuevas redes a las que se integran algunos actores anteriores. En esas nuevas formaciones, nuevos contactos concentran la centralidad y la intermediación, lo que muestra un reemplazo de roles y funciones.

\section{Actores y sus características en redes de hombres antes de enviudar}

Las redes sociales de los casos de hombres revisadas muestran que, en el periodo antes de enviudar, estas estaban formadas mayoritariamente por amigos y amigas y por compañeros de trabajo. Si bien la familia estaba presente en ellas, este vínculo se observa en menor medida que en el resto de los actores que conforman estas redes.

En términos de género, las redes previudez que incluyen mayor cantidad de amigos y de personas con las que compartían algún tipo de actividad, es decir, compañeros, están compuestas principalmente por hombres; y en los casos en los que hay mayor presencia de mujeres, estas corresponden a aquellas que son de tipo más familiar, ocupando tanto una función y un rol de mayor importancia que los hombres.

Finalmente, existen algunas redes sociales que incluyen otro tipo de actores femeninos identificados como vecinas, comadres y empleadas; destaca la importancia de estos dos últimos actores en la formación de la red, pues se ubican en su interior junto a otros actores influyentes.

\section{Actores y sus características en redes de hombres después de enviudar}

En las redes sociales analizadas posteriores al hito de la viudez, se advierte una reducción del número de integrantes producto del fallecimiento de algunos de ellos. Pese a eso, el número total de actores que constituyen las redes posviudez se mantiene como en el periodo anterior, puesto que los viudos tienden a integrar nuevos actores a su red.

La red posviudez se mantiene, ya que, si bien han incluido nuevos actores a la misma, la estructura principal no cambia. No obstante, se advierten nuevas características en ella.

En algunos casos se observa en la red posviudez la integración de actores que previamente no establecían vínculos entre sí. En el periodo posviudez esos actores se relacionan entre sí, producto de la presencia de algunos contactos claves que se van uniendo hasta configurarse como una sola estructura. Este es el caso, por ejemplo, de una red previudez formada por tres subestructuras y en que, luego de enviudar, uno

\section{MUJER}

FIGURA № 2: “Configuración de red social en mujer antes y después de enviudar, según centralidad”
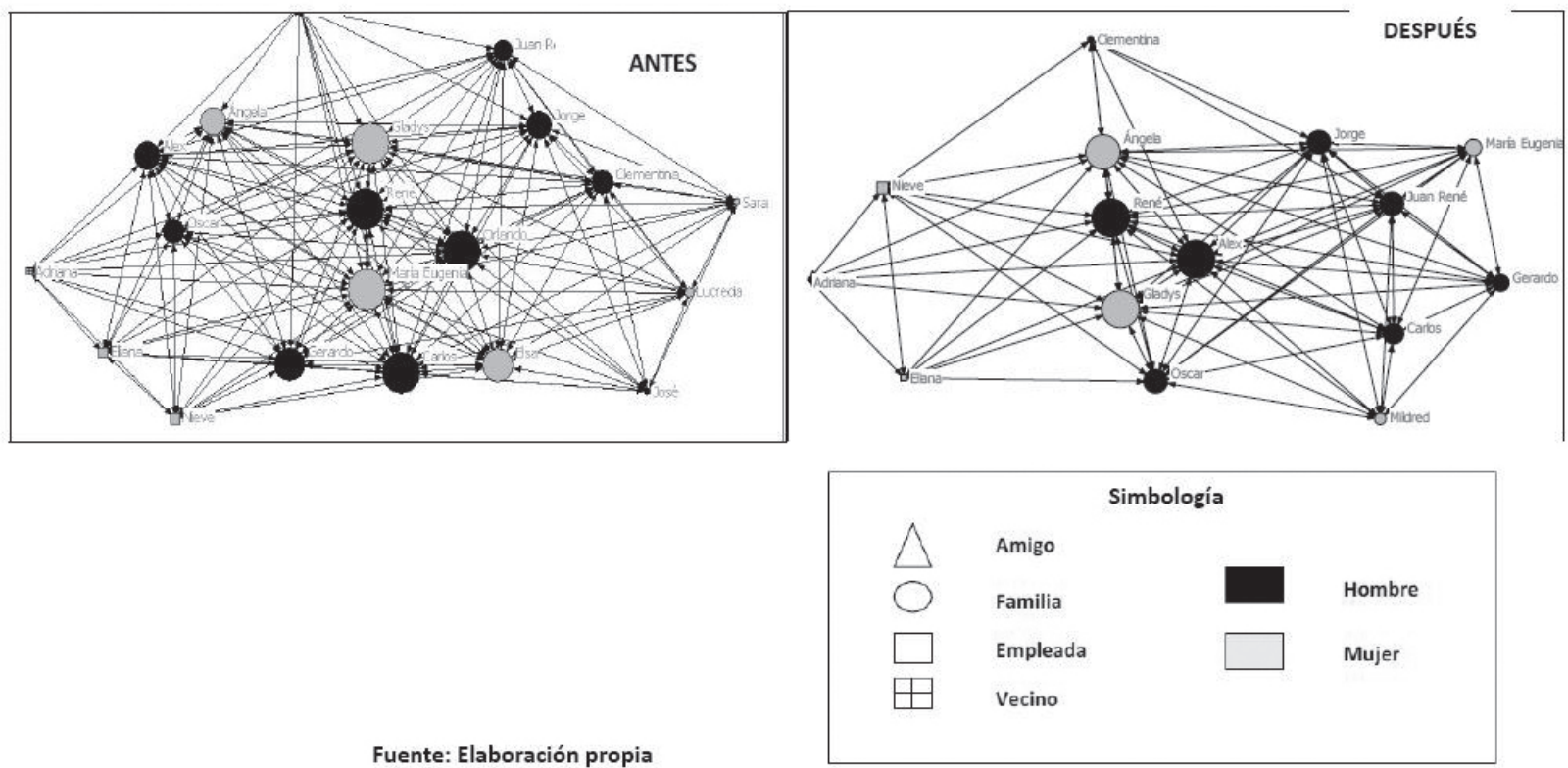
de los actores con tipo de vínculo amigo asume el rol y la función de conectar y ser puente de los miembros de las otras subestructuras, configurándose así una sola estructura en la red posviudez (véase la figura 3 ).

La estructura de la red social también se puede mantener en la configuración de la red previudez. En ellas, los actores claves - principalmente la cónyugeson reemplazados por otros contactos de la misma red -generalmente los hijos e hijas-, cumpliendo un nuevo rol y función. En estas redes la falta de la esposa no afecta la configuración de la red, salvo el caso de actores específicos de vínculo amigo, que pierden relación con la red, quedando totalmente aislados de ella.

Aun cuando la estructura principal de la red previudez tiende a mantenerse, en los casos estudiados se observan la incorporación de nuevos actores a la red posviudez. Estos nuevos actores incorporados tienen diferentes características dependiendo de cada caso, pero destaca que todos los viudos entrevistados incluyen nuevos contactos a su red. De esta manera algunos de los casos analizados presentan más actores considerados como amigos y amigas y, a la vez, menos actores identificados como compañeros; esos casos muestran que el entrevistado forma vínculos más cercanos con los miembros de su red postviudez. En otros casos se observa mayor presencia de actores nuevos de tipo vínculo compañeros y compañeras, lo que da cuenta de que el entrevistado, después de la viudez, empezó a realizar otras actividades donde conoció a nuevas personas y contactos que se van haciendo parte de su red después de enviudar; generalmente esas actividades corresponden a participación en clubes o asociaciones de personas mayores. Tam- bién hay casos donde se observa mayor interacción con el barrio donde se reside, lo que se traduce en la inclusión a la red social de nuevos actores de tipo vínculo vecinos.

En algunas redes sociales se observa que la incorporación de nuevos actores da paso a la formación de nuevas subestructuras en el interior de la red social, es decir, que mantienen vínculos entre sí (cliques), pero que no establecen vínculos con la estructura principal, la cual, como se mencionó, se mantiene en la red previudez (véase la figura 4).

\section{Propiedades estructurales de las redes sociales de hombres}

En el análisis de los viudos se observaron las características de las redes, los niveles de integración, la presencia de subestructuras y los niveles de influencia de algunos de los actores que las forman.

Las redes sociales previudez de los hombres corresponden a redes densas ya que sus miembros mantienen vínculos entre sí o pueden acceder a otro $u$ otros contactos por medio de algún actor influyente; no advirtiéndose contactos aislados de la red. Destacan en las redes previudez la presencia de actores claves, lo que se observa en las medidas de centralidad y de intermediación que presentan. De las redes sociales revisadas, los actores que se identifican como actores poderosos corresponden a familiares de tipo directo donde destaca la figura del cónyuge, también personas cercanas consideradas como comadre o personas presentes en la cotidianeidad del viudo, como el caso de la figura de la empleada.

\section{HOMBRE}

FIGURA № 3: "Configuración de red social en hombre antes y después de enviudar"
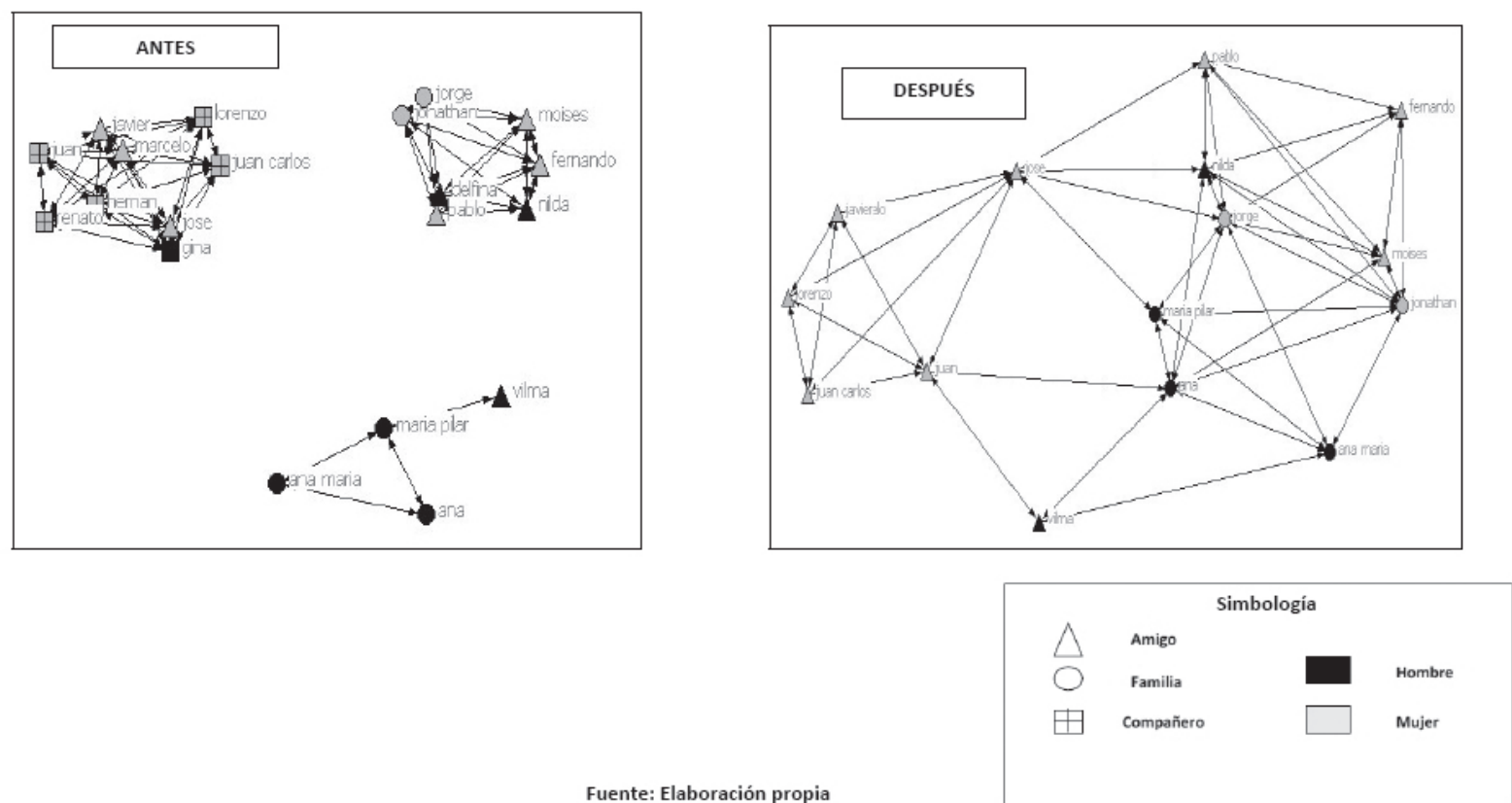


\section{HOMBRE}

FIGURA № 4: "Configuración de red social en hombre antes y después de enviudar, según centralidad"
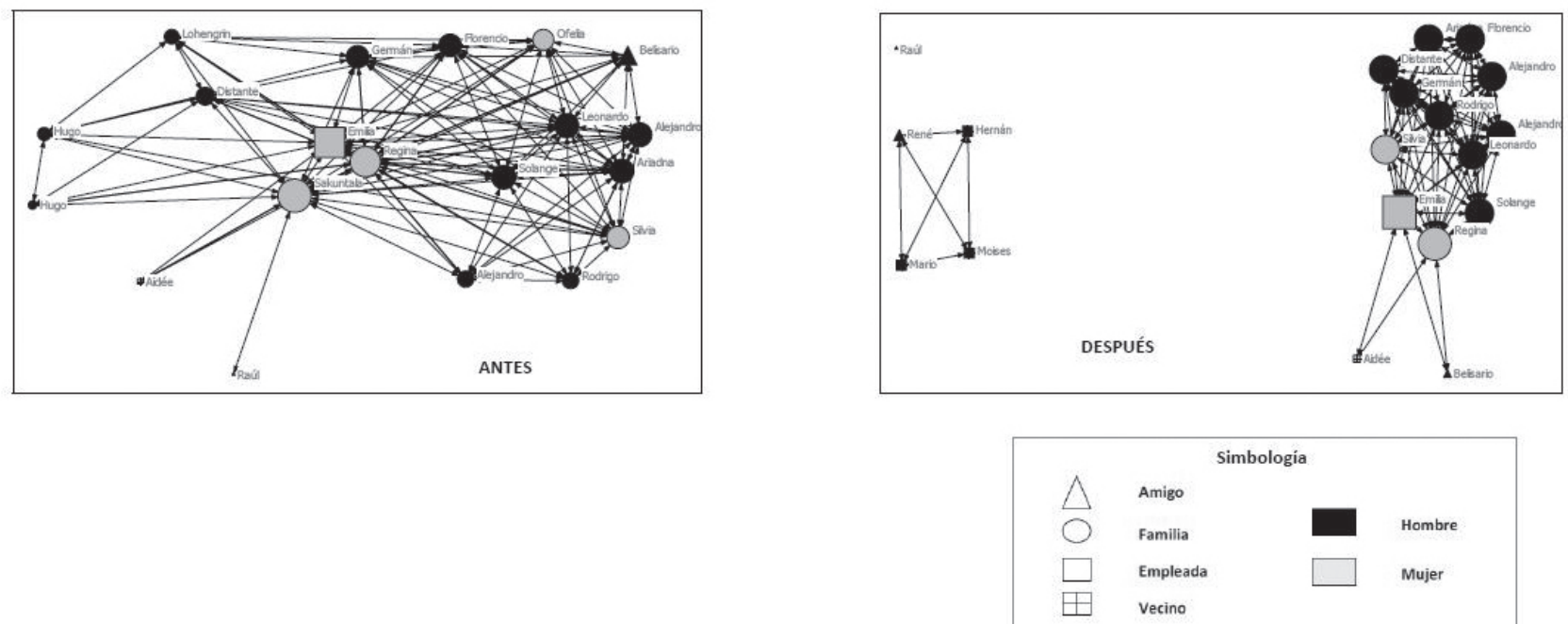

En la morfología de las redes posviudez, las estructuras principales se mantienen; esto se manifiesta en que la densidad no cambia o en algunos casos sube (por ejemplo 0,5 antes y 0,8 después de enviudar), lo que indica que los miembros de la red social siguen estableciendo vínculos entre sí. No obstante, las medidas de centralidad y de intermediación varían (por ejemplo, en centralidad, $17,6 \%$ a $45 \%$; e intermediación, $46,2 \%$ a $33,6 \%$ ), producto principalmente de la ausencia de la cónyuge, quien ocupaba una posición relevante en la red previudez; esa posición es asumida por otros actores presentes en esa red, pero que en la red posviudez asumen otras funciones y roles; como por ejemplo el caso de la hija que después de fallecer la madre toma su lugar. De acuerdo a esto, si bien los viudos incorporan nuevos actores a la red social, esos nuevos actores no ocupan, necesariamente, posiciones influyentes en la red.

\section{Discusión}

El análisis e interpretación de los cambios presentados en la configuración de las redes de relaciones sociales, tanto en hombres como en mujeres después de enviudar, nos permiten posicionar su vida conyugal como un evento biográfico significativo, y la muerte del cónyuge como un hito; cómo una situación social y vital se constituye en una referencia significativa a la hora de observar o evaluar la posición de la persona mayor viuda en su universo de relaciones y vinculaciones sociales. La viudez como hito en la configuración de redes sociales es una experiencia que llega a desencadenar nuevas relaciones significativas; es un evento destacable, emblemático, ya sea en términos positivos o negativos, pues las relaciones no solo pueden aumentar, sino también disminuir por la muerte de algunos de los actores que componen la red. La viudez marca, separa o activa la reorganización de los lazos sociales y el sentido de la vida (Guiax et al., 2007; Patterson y Carpenter, 1994). Si afirmamos que la viudez es una experiencia dinámica, que, por un lado, sobresale en las características de las redes y sus morfologías y, por otro lado, es un eje desencadenante de otros, podemos identificar y construir diversos tipos de redes de relaciones sociales en hombres y mujeres viudas.

La información empírica nos ha mostrado que para el caso de las mujeres es posible identificar tres tipos de configuración de la red:

A. Red que se mantiene, principalmente compuesta por vínculos familiares, ya sean del tipo familiar par, familiar descendiente o familiar ascendente; en ella, los actores nuevos de la red reemplazan los desaparecidos por fallecimiento, donde, el vínculo familiar par o ascendente es reemplazado por actores de vínculo descendiente, apareciendo con fuerza la figura de la hija o de el hijo que se va a vivir con la madre viuda. El rol gravitante que asumía el cónyuge lo asume este familiar descendiente, manteniéndose la morfología de la red, su tamaño y componentes de vínculo familiar.

B. Red que cambia y se reconfigura, donde los vínculos de tipo familiar son reemplazados por actores de vínculo compañeros. Si bien el tamaño es el mismo, los componentes de la red y sus relaciones cambian, dándole otra morfología a la misma. El contexto social de estas viudas cambia con la muerte del cónyu- 
ge, siendo principalmente de participación y de integración en actividades comunitarias. Representan a la "viuda liberada" que comienza a realizar una serie de actividades nuevas en la viudez. En la configuración de la red posviudez, prácticamente desaparecen los actores de vínculo familia y se reconfigura con referentes significativos fuera del espacio doméstico y familiar.

C. Red que se desintegra adquiriendo una morfología atomística, donde la debilidad de la red, después de la muerte del cónyuge, se manifiesta en el reducido contacto que tienen los componentes entre sí; llegando a constituirse tres subestructuras, en las cuales ninguno de sus miembros se relacionan con los de las otras. El vínculo más significativo para la viuda llega a ser el de los compañeros, pues sus actores están presentes en todas las subestructuras. El vínculo familia sigue siendo el predominante en cuanto a número de elementos que componen la red.

En el caso de los hombres se identifican cuatro tipos de configuración de la red:

A. Red que se mantiene, al igual que en las mujeres viudas, la cual ocurre principalmente en los casos en que un vínculo familiar descendiente-como puede ser la hija- o par asume un rol importante en las relaciones sociales de su padre, con otros vínculos, como pueden ser los del tipo amigos. Después de enviudar, la red se caracteriza por el mantenimiento también de la presencia de vínculo tanto familia como amigos; lo que da cuenta de cambios poco significativos en la vida cotidiana y las relaciones interpersonales de estos hombres después de la muerte de la esposa.

B. Red que se integra. A diferencia de las mujeres, esta configuración se caracteriza por la integración de dos tipos de vínculos que antes de la muerte de la esposa conformaban una red con subestructuras no relacionadas entre sí. El vínculo familia y el vínculo amigos comienzan a relacionarse, dando forma a una sola estructura de red. Algunos hombres después de enviudar disminuyen el desarrollo de actividades fuera del hogar, y el vínculo compañeros de su red tiende a desaparecer.

C. Red que cambia en el sentido de que la estructura principal se reconfigura y sigue existiendo, pero se hace mucho más densa por la intensificación de los contactos entre sus miembros, lo cual, a la vez, la hace una red más fuerte para el viudo.

D. Red que cambia con creación de subestructuras. En este último tipo, si bien la red cambia, incorpora subestructura(s) en su configuración, así, en la red posviudez aparecen actores asociados a vínculos del tipo compañeros y vecinos.

\section{Conclusiones}

Comprender la muerte del cónyuge como un hito biográfico-social nos ha planteado un cambio significativo en el proceso de envejecimiento en las personas. Pero ¿qué significa que la red social pueda tender al aumento o disminución una vez que se ha enviudado?

Por el contrario lo que nos hemos encontrado es que la cantidad de nodos, el número, se mantiene antes y después de la viudez, lo que puede tener que ver con la biografía de cada uno. Las personas mantienen el número de contactos con los que están acostumbrados a vincularse, reemplazándolos cuando van desapareciendo, pero manteniendo prácticamente la misma cantidad de vinculaciones posibles. No obstante, la configuración de la red cambia, pudiendo identificarse diversos tipos tanto en las redes de los hombres como de las mujeres viudas. Esto nos estaría mostrando la importancia que tiene el cónyuge, en estas generaciones, para la vinculación entre los miembros de la red; a lo largo de sus trayectorias conyugales, tanto hombres como mujeres van construyendo vínculos sociales donde el esposo o esposa juega un rol relevante.

La viudez en la vejez se manifiesta al observar las redes de relaciones sociales, y se constituye en un hito biográfico que marca un cambio, un punto de inflexión en la configuración de la red social, específicamente referida al acceso y vínculos de los miembros de la red entre sí, y su influencia en la misma. De esta forma el hito o evento biográfico que encierra la viudez puede llevar a modificar el tipo de vínculo y actores, pero no necesariamente el tamaño de la red, situación que puede llevar a concebir a la red social con una función de coherencia y continuidad biográfica en el curso de la vida.

Analizar la configuración de las redes de hombres y mujeres antes y después de enviudar nos permite entender a la viudez como un hito en el continuum biográfico del envejecimiento y a las redes mismas como una articulación en la vida de las y los envejecidos, vale decir, una realidad que orienta la 
experiencia vital y la construcción de la memoria individual y social.

\section{Referencias}

ARBER, S.; GINN, J. Opiniones y limitaciones de las mujeres casadas ante la jubilación. In: (Coord.). Relación entre género y envejecimiento. Madrid: Narcea, 1996. p. 105-126.

ARNOLD, M. et al. Inclusión y exclusión social del adulto mayor en Chile. Opiniones, expectativas y evaluaciones de la población chilena sobre diferentes modalidades de inclusión $y$ exclusión social de las personas adultas mayores. Santiago: Programa de Estudios Sistémicos y Fondo de Población de las Naciones Unidas. 2009. Disponible en: <http://www. esistemicosvejez.cl/upload/est_pdf/7.pdf>.

ASKHAM, J. Vida matrimonial de las personas mayores. In: ARBER, S.; GINN, J. (Coord.). Relación entre género y envejecimiento. Madrid: Narcea, 1996. p. 127-140.

BAZO, M. T. La institución social de la jubilación: de la sociedad industrial a la postmodernidad. Valencia: Nau Llibres, 2001.

BERARDO, F. M. Survivorship and social isolation: the case of the aged widower. The Family Coordinator, v. 19, p. 11-25, 1970 .

BERNARD, M. et al. Trabajo y jubilación marcados por el género. In: ARBER, S.; GINN, J. (Coord.). Relación entre género y envejecimiento. Madrid: Narcea, 1996. p. 89-104.

BUITELAAR, M. "Widows" worlds: representations and realities. In: BREMMER, J.; VAN DEN BOSCH, L. (Eds.). Between poverty and the pyre: moments in the history of widowhood. London: Routledge, 1995. p. 1-18.

DAVIDSON, K. Late life widowhood, selfishness and new partnership choices: a gendered perspective. Ageing and Society, v. 21, p. 297-317, 2001.

DEL VALLE, T. (Coord.). Modelos emergentes en los sistemas $y$ las relaciones de género. Madrid: Narcea, 2002.

FERRARO, K. F. Widowhood and social participation in later life. Research on Aging, v. 6, n. 4, p. 451-468, 1984. GUIAX, M. et al. Changes in contact and support exchange in personal networks after widowhood. Personal Relationships, v. 14, p. 457-473, 2007.

GUTIÉRREZ, E.; Ríos, P. Envejecimiento y campo de la edad: elementos sobre la pertinencia del conocimiento gerontológico. Revista Última Década, v. 25, p. 11-41, 2006. HERRERO, R.; SÁNCHEZ, M. La terminología del análisis de redes. Problemas de definición y de traducción. Política y Sociedad, v. 33, p. 199-206, 2000.
INE. Censo 2002. Santiago: Instituto Nacional de Estadística, 2002.

INE. Enfoque estadístico: adulto mayor en Chile. Disponible en: <http://www.ine.cl/canales/sala_prensa/ noticias/2007/septiembre/boletin/ine_adulto_mayor. pdf>. 2007. Acceso en: 10 fev. 2012.

INGRISCH, D. Adaptación y resistencia de las mujeres a medida que envejecen. In: ARBER, S.; GINN, J. (Coord.). Relación entre género y envejecimiento. Madrid: Narcea, 1996. p. 71-88.

MCCALLUM, J. Retirement and widowhood transitions. In: KENDIG, H. (Ed.). Ageing and families: a support networks perspective. Sydney: Allen \& Unwin, 1986.

MCLAUGHLIN, D. et al. Factors which enhance or inhibit social support: a mixed-methods analysis of social networks in older women. Ageing \& Society, v. 31, p. 1833, 2011.

MOEN, P.; SPENCER, D. Converging divergences in age, gender, health, and well-being: strategic selection in the third age. In: BINSTOCK, R.; GEORGE, L. (Ed.). Handbook of aging and social sciences. California: Academic Press, Elsevier, 2006.

OSORIO, P. La longevidad más allá de la biología. Aspectos socioculturales. Papeles del CEIC, v. 22, p. 1-28, 2006. Disponible en: <http://www.ehu.es/CEIC/pdf/22. pdf>. Acceso en: 20 fev. 2012.

PATTERSON, I.; CARPENTER, G. Participation in leisure activities after the death of a spouse. Leisure Sciences, v. 16, n. 2, p. 105-117, 1994.

RIGGS, A. Men, friends and widowhood: toward successful ageing. Australian Journal on Ageing, v. 16, n. 3, p. 182-185, 1997.

ROSE, H.; BRUCE, E. Diferente valoración de la ayuda que se prestan las personas ancianas. In: ARBER, S.; GINN, J. (Coord.). Relación entre género y envejecimiento. Madrid: Narcea, 1996. p. 163-182.

SCOTT, A.; WENGER, G. C. Género y redes de apoyo social en la vejez. In: ARBER, S.; GINN, J. (Coord.). Relación entre género y envejecimiento. Madrid: Narcea, 1996. p. 221-240.

SWANN, A. Men and bereavement Nursing (London). Journal of Clinical Practice, Education and Managemen, v. 3, n. 26, p. 966-968, 1988.

WILSON, G. Yo soy los ojos y ella los brazos: cambios en los roles de género en la vejez avanzada. In: ARBER, S.; GINN, J. (Coord.). Relación entre género y envejecimiento. Madrid: Narcea, 1996. p. 141-162.

WISTER, A.; STRAIN, L. Social support and wellbeing: a comparison of older widows and widowers. Canadian Journal on Aging, v. 5, n. 3, p. 205-220, 1986. 


\title{
Configuration of social networks in widowed older people in Chile
}

\begin{abstract}
This article presents the results of a study on the configuration of social networks of widowed people aged 60 and more in the Metropolitan Region of Santiago, Chile. This work emphasizes the conjunction between the structuring field of aging and the life experiences of older people. Through a research focused on the aging processes in Chile, using social network analysis, the morphology of the networks and the types of links of older people before and after becoming widowed are observed, as well as the way they affect the social construction of aging. More precisely, the latter refers as the identity of the older individual is constructed and re-constructed when the dead of the spouse has the meaning of a life transition. Social relationships, their formation, experiences, and meaning are the axis to the analysis and comprehension of the social phenomenon of widowhood in later life.
\end{abstract}

Key words: old age, widowhood, social relationships, life transition.

\section{Configurações de redes sociais em viúvos idosos no Chile}

\section{Resumo}

O artigo apresenta os resultados de uma pesquisa sobre a configuração de redes de relações sociais com viúvos de mais de 60 anos na região metropolitana de Santiago, no Chile. Além disso, aponta para a conjunção entre a estruturação do campo da velhice e as experiências vitais dos idosos. Através de uma pesquisa delimitada pelos processos de envelhecimento no Chile, observam-se a morfologia das redes e os tipos de vínculos dessas pessoas, antes e depois da viuvez, e como esse fato influencia a construção social da velhice. Enfim, como se constrói e reconstrói a identidade do idoso ao entender a morte do cônjuge como um marco biográfico-social, sendo as relações interpessoais, sua construção, experiência e significações os eixos para a análise e a compreensão do fenômeno social da viuvez na velhice.

Palavras-chave: velhice, viuvez, relações interpessoais, transições biográficas.

Data de recebimento do artigo: 9/12/2013

Data de aprovação do artigo: 6/8/2014 